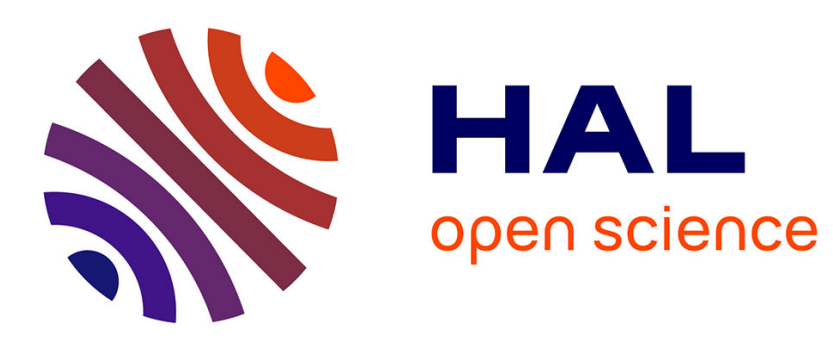

\title{
A coupled BEM and energy flow method for mid-high frequency internal acoustic
}

Sébastien Besset, Mohamed Ichchou, Louis Jezequel

\section{To cite this version:}

Sébastien Besset, Mohamed Ichchou, Louis Jezequel. A coupled BEM and energy flow method for mid-high frequency internal acoustic. Journal of Computational Acoustics, 2010, 18 (1), pp.69-85. 10.1142/S0218396X10004073 . hal-00625137

\section{HAL Id: hal-00625137 \\ https://hal.science/hal-00625137}

Submitted on 22 Sep 2011

HAL is a multi-disciplinary open access archive for the deposit and dissemination of scientific research documents, whether they are published or not. The documents may come from teaching and research institutions in France or abroad, or from public or private research centers.
L'archive ouverte pluridisciplinaire HAL, est destinée au dépôt et à la diffusion de documents scientifiques de niveau recherche, publiés ou non, émanant des établissements d'enseignement et de recherche français ou étrangers, des laboratoires publics ou privés. 


\title{
A coupled BEM and energy flow method for mid-high frequency internal acoustic
}

\author{
Sbastien BESSET, M.N. Ichchou and L. Jézéquel \\ LTDS/D2S, École Centrale de Lyon, \\ 36 av. Guy de Collongue, 69134 Ecully cedex, France \\ Journal of Computational Acoustic, 18 (1), 69-85, 2010
}

\begin{abstract}
This paper deals with the behaviour of acoustic cavities in the mid-high frequency range. The method proposed here is based on an energy flow method named Simplified Energy Method (MES). MES method is quite efficient in the mid-high frequency range but the directivity of the boundary sources is not well estimated. We propose a hybrid method which couples MES and the Boundary Element Method (BEM). Thus, the BEM method is used to estimate the direct field, considering a "correct" directivity. As a complete calculation is not adapted to BEM in mid-high frequency range because of the calculation costs, we only apply BEM on the domains including boundary sources. Other parts of the system and the reverberated field are estimated by the mean of MES method. This hybrid method leads to a consistent prediction of injected power densities. Numerical comparisons prove the efficiency of the proposed reformulation.
\end{abstract}

keywords: Energy flow; BEM; Energy method; Injected power density; Energy density; Intensity.

\section{Introduction}

Medium and high frequency range is a fundamental topic of research in the context of viroacoustics. In fact, well established and deeply studied numerical methods like finite element or boundary equation based formulations run into important limitations as frequency increases. The most important limitation is the size of the meshing that must be very fine and leads to high calculation costs. Modal methods base on finite element formulations have been develop [1, 2], but the calculation costs remain high and these methods are limited to medium frequency range.

Among possible alternatives, analysis methods which are energy variables based are often studied. The Statistical Energy Analysis (SEA) [3] is the recognized mother and gives the mechanical energy of complex buildup structures. However SEA suffers from its axiomatic formulation and still requires improvements. Beyond these studies, a number of works attempts to enhance the Statistical Energy Analysis robustness and 
predictivity. Among those tentatives, lets report the earlier work of Nefske and Sung [4] who proposed the use of an energy diffusion model to predict the space spread of energy density within subsystems. Basically, this way of thinking can be viewed as a local energy formalism whereas the SEA formalism is based on global energies of finite subsystems. This model has been improved in [5, 6, 7, 8, 9, 10]. Among other contributors to this subject, let mention references [11, 12, 13, 14] where interesting discussions are given. In [8, 9, 10] energy models started with the so-called general energy method. The main goal of this method was to reformulate the classical displacement models using four energy variables: the total energy as well as the Lagrangian energy density, the active and the reactive energy flow. From the elimination of the Lagrangian energy density and the reactive energy flow, this general energy formulation leads to an interesting energy model well suited for medium and high frequency dynamics. In fact, from a propagative approach, it has been shown that the Lagrangian energy density and the reactive energy flow is mainly linked to wave interferences or singularities. So that, taking into account only the incoherent contribution of waves to the energy variables leads to the formulation of the local energy approach concerned here. References [9, 10, 15, 16, 17] give in depth the formulations used in the context of local energy approach applications. The MES has already been applied to various domains: beams [8, 18], membranes and plates [19, 20, 11, 8, 15] and acoustical radiation [21, 22, 18]. In this paper, the main concern is the improvement of MES prediction for internal acoustics [23].

The main addressed issue in this work is the injected power density for use in the MES formulation. The injected power density is usually described from the input acoustic mobility. The later is easily expressed for unbounded media. A major concern in the MES prediction quality is the input source directivity which needs to be fixed. So the major question this paper tries to solve is the concise estimation of injected power density. So far, the MES energy method needs an estimation of the entering power, and its directivity, which cannot easily be estimated and is usually considered to be lambertian. This is an assumption that is not always verified and the proposed hybrid method aims at estimating correctly this directivity. Particularly, the computation of the direct field is the key aspect. A coupled BEM and MES strategy is chosen. The algebraic coupled formulation is expressed and implemented. Numerical experiments was achieved comparing the coupled strategy with the conventional one. The interest and the feasibility of the finding is proved. It must be noticed here that authors already studied the coupling between deterministic and statistical subsystems. Among them, Shorter and Langley [24] considered the coupling between several subsystems, each subsystem being described with deterministic or statistical methods. This work has lead to coupling methods that are currently used in softwares as VAOne. Nevertheless, the method we propose does not deal with coupling substructures, but allows to describe a single structure considering a mixed formulation.

First, the Simplified Energy Method is recalled. The hybrid formulation is then presented. Principle of the method is given and a matrix formulation is proposed. At last, numerical calculations are processed to show the efficiency of the method. 


\section{Simplified Energy Method (MES)}

\subsection{Assumptions}

In view of describing the energy transfer inside the medium, two continuous energy fields are introduced. The first energy quantity is nothing else than the total energy density $W(\overrightarrow{\mathbf{s}}, t)$ defined as the sum of the potential energy density and the kinetic energy density. The second energy variable $\overrightarrow{\mathbf{I}}(\overrightarrow{\mathbf{s}}, t)$ is the energy flow. These quantities are local in opposition with energies per subsystem involved in SEA. The energy balance equation from continuum mechanics obeying conservation principle, which govern the energy density in various vibrating system can be described by:

$$
\frac{\partial W}{\partial t}=-\pi_{\mathrm{diss}}-\vec{\nabla} \cdot \vec{I}
$$

where $\vec{\nabla}$ is the gradient operator, $\pi_{\text {diss }}$ is the power density dissipated. The damping model adopted here is the same as in SEA (power density being dissipated is proportional to the energy density). Hence:

$$
\pi_{\text {diss }}(\overrightarrow{\mathbf{s}})=\eta \omega W(\overrightarrow{\mathbf{s}})
$$

where $\eta$ is the damping loss factor and $\omega$ the circular frequency. The validity of this relationship has been discussed in the literature [3] about SEA. Equation (1]) is the energy balance relation for all elastoacoustic media and it is regarded as a valid method for steady or transient analysis. In order to derive the energy density equations, a wave description of vibrational-acoustical behaviour is definitely adopted. In the subsequent presentation planes and symmetrical propagating disturbances in a medium are considered.

Each propagating waves involve partial energy quantities defined as the energy variables associated to those waves. In order to establish a relationship between the partial energies $W_{i}(\overrightarrow{\mathbf{s}})$ and $\overrightarrow{\mathbf{I}}_{i}(\overrightarrow{\mathbf{s}})$ and the total ones $W(\overrightarrow{\mathbf{s}}), \overrightarrow{\mathbf{I}}(\overrightarrow{\mathbf{s}})$, an additional assumption is introduced. In fact, a superposition principle is assumed and applied here for all quadratic variables:

$$
£(\overrightarrow{\mathbf{s}})=\sum_{i} £_{i}(\overrightarrow{\mathbf{s}})
$$

$£, £_{i}$ being a quadratic variables, representing either a partial energy or a global energy associated to a particular wave field. This assumption is often used in statistical phenomenon in physics. We can find such an assumption in room acoustics, electromagnetism,... In the following developments an intrinsic energy law will be used. In fact, this superposition principle is valid if a term due to the phase is neglected. Considering two waves denoted $v_{1}$ and $v_{2}$, we can write the following equation:

$$
\left|u_{1}+u_{2}\right|^{2}=\left|u_{1}\right|^{2}+\left|u_{2}\right|^{2}+\underbrace{u_{1}^{*} u_{2}+u_{1} u_{2}^{*}}_{\text {neglected terms }}
$$

These terms can be neglected because the quantities considered in MES are frequency average quantities. Therefore, we can write: 


$$
<u_{1}^{*} u_{2}>=0 \quad \text { and } \quad<u_{1} u_{2}^{*}>=0
$$

where $\langle u . v\rangle=\frac{1}{\Delta f} \int_{\Delta f} u . v d f, \Delta f$ being the considered frequency range for the frequency average. This will mean that MES does not take into account modal information (linked to the phase of the waves).

The following equation [25, 26] is often introduced in order to define the wave velocity $c$. In fact:

$$
\vec{I}^{i}(\overrightarrow{\mathbf{s}})=c \cdot W^{i}(\overrightarrow{\mathbf{s}}) \overrightarrow{\mathbf{n}}
$$

This expression was shown to be a valid law for non-damped waves field.

\subsection{Elementary fields: planes waves}

In this section, a wave approach very similar to the one given in [27], will be used. Hence, expression (6) will be first introduced in the energy flow balance (1), leading to:

$$
c \vec{\nabla} \cdot W(\overrightarrow{\mathbf{s}}) \overrightarrow{\mathbf{n}}+\eta \omega W(\overrightarrow{\mathbf{s}})=0
$$

Equations 6 and 7 lead then to the following relation:

$$
c \vec{\nabla} W(\overrightarrow{\mathbf{s}}) \overrightarrow{\mathbf{n}} \cdot \overrightarrow{\mathbf{n}}+\frac{\eta \omega}{c} \vec{I}(\overrightarrow{\mathbf{s}})=0
$$

Thus, it can be readily shown using planes waves characteristics that the total energy density and the active energy flow are linked by:

$$
\begin{gathered}
-c^{2} \vec{\nabla} W(\overrightarrow{\mathbf{s}})=\eta \omega \vec{I}(\overrightarrow{\mathbf{s}}) \\
\Delta W(\overrightarrow{\mathbf{s}})-\eta^{2} \omega^{2} W(\overrightarrow{\mathbf{s}})=0
\end{gathered}
$$

where $\Delta=\vec{\nabla} \cdot \vec{\nabla}$. This is the local energy equation for an elementary planes waves field. This is an hyperbolic equation of telephone format which reduces to the simple wave equation when no dissipation mechanics is introduced. This equation needs energy boundary conditions and coupling conditions to be defined.

\subsection{Elementary fields: symmetrical waves}

Let us consider now a symmetrical waves field. Namely, we will consider cylindrical waves in dimension 2, and spherical waves in dimension 3. The notations used in this section are recalled on figure $1 .\left(\vec{I}_{d}, W_{d}\right)$ is the direct field whereas $\left(\vec{I}_{r}, W_{r}\right)$ is the reverberated field. $\vec{n}$ is the unit normal vector at boundary. In this cases, combining (6) and (1) leads to:

$$
\frac{1}{r^{n-1}} \frac{\partial}{\partial r}\left(r^{n-1} \vec{I}\right)+\eta \omega W \overrightarrow{\mathbf{n}}=0
$$

using (11), one can readily show that:

$$
-c^{2} \frac{1}{r^{n-1}} \frac{\partial}{\partial r}\left(r^{n-1} W \vec{n}\right)=\eta \omega \vec{I}
$$




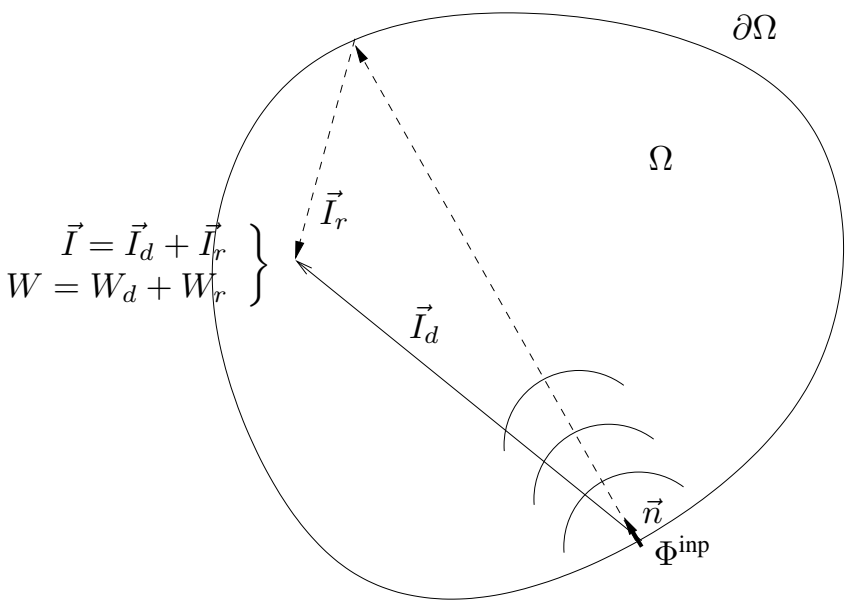

Figure 1: Notations used in section 2.3

Putting this intrinsic law in the energy flow balance (1) leads to:

$$
\frac{1}{r^{n-1}} \frac{\partial}{\partial r}\left(r^{n-1} W\right)-\eta^{2} \omega^{2} W=0
$$

This is the local energy equation for symmetrical waves.

Figure 2 shows two distinguish manner of energy reconstruction. In both techniques, energy variables are defined as the supperposition of a direct field and a reverberant field. In both techniques, the direct field is calculated assuming a symmetrical waves fields like described before. The difference comes from the reverberant field treatment.

$$
W=W_{d}+W_{r}
$$

and

$$
\overrightarrow{\mathbf{I}}=\overrightarrow{\mathbf{I}}_{d}+\overrightarrow{\mathbf{I}}_{r}
$$

Precisely, in figure 2 2 a), the reverberant field is assumed to be a set of planes waves. This approach is known as the hybrid energy flow method. Here we will designate it by the mixed wave solution. This solution was proposed by Smith [28]. Further developments and considerations are given in [29]. The second approach considers the reverberant filed as the results of secondary sources located at boundaries as shown in figure 2 b). So, only symmetrical waves fields is considered. This solution was first proposed by Kuttruff [30, 31, 32, 33]. It is also known as the radiosity method. Furthers contributions and details can be found in [34, 35] and [36]. It is this second approach that we will use in this paper.

Here, both the direct and the reverberant field will be represented by symmetrical waves. The elementary solutions in terms of energy density and active intensity will be denoted $G$ and $\vec{H}$.

$$
G(\overrightarrow{P M})=\frac{1}{\gamma_{0} c} \frac{e^{-\eta \omega r}}{r^{n-1}} \quad \vec{H}(\overrightarrow{P M})=\frac{1}{\gamma_{0}} \frac{e^{-\eta \omega r}}{r^{n-1}} \vec{u}_{r}
$$


(a)

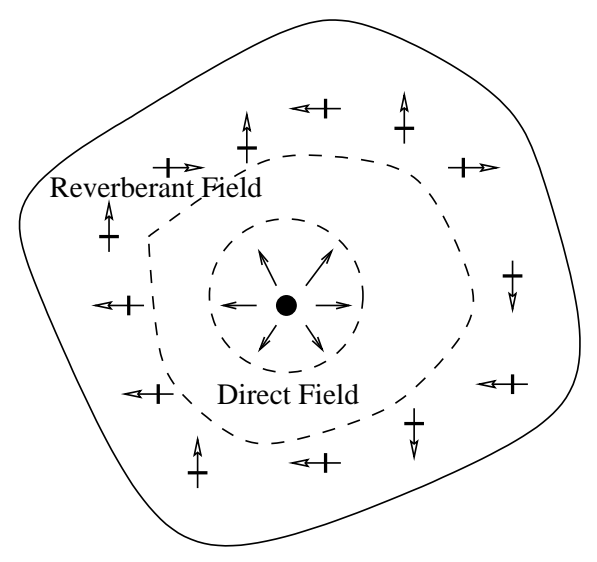

(b)

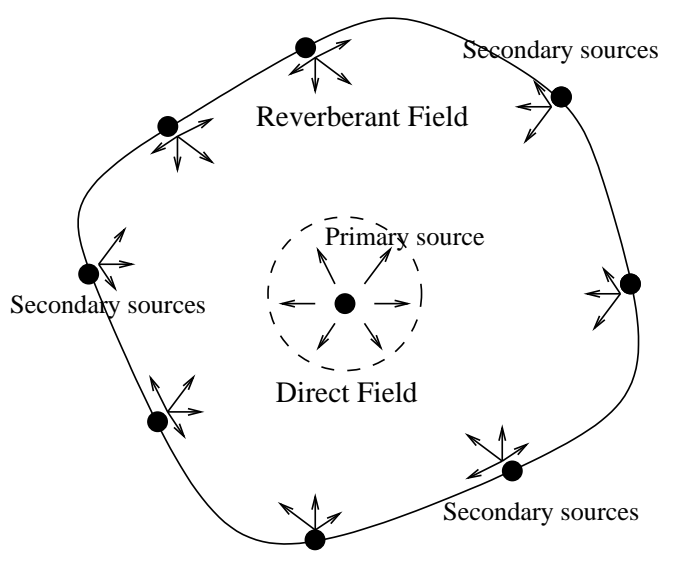

Figure 2: Energy fields reconstruction: (a) mixed wave assumption and (b) fictitious sources at boundaries.

where $\vec{u}_{r}=\frac{\overrightarrow{P M}}{\|\overrightarrow{P M}\|}$ and $r=\|\overrightarrow{P M}\|$. Hence, using a superposition principle the energy solution is then expressed using the contribution of the primary source (direct field) and the contribution of the fictitious sources. The fictitious sources are energy unknown parameters representing the contribution of the reverberant filed. Precisely:

$$
W(M)=\int_{\Omega} \rho(P) G(\overrightarrow{P M}) d P+\int_{\partial \Omega} \sigma(P) \vec{u}_{r} \cdot \vec{n} G(\overrightarrow{P M}) d P
$$

The power flow can be described by a similar expression:

$$
\vec{I}(M)=\int_{\Omega} \rho(P) \vec{H}(\overrightarrow{P M}) d P+\int_{\partial \Omega} \sigma(P) \overrightarrow{u_{\overrightarrow{P M}}} \cdot \vec{n} \vec{H}(\overrightarrow{P M}) d P
$$

where $\rho(P)$ corresponds to volumic sources and $\sigma(P)$ corresponds to surfacic sources. Energy variables are given as a solution of a Fredholm equation, corresponding to an energy balance at the boundary of the domain [30].

\subsection{Discretized formulation}

Let us consider an isolated system as shown in figure 3 .

The boundaries of the cavity are discretized ( $N_{e}$ elements). Properties are given for each element $i$ of the meshing:

- $\Phi_{i}^{\mathrm{inj}}(\omega)$ is the input power flow; 


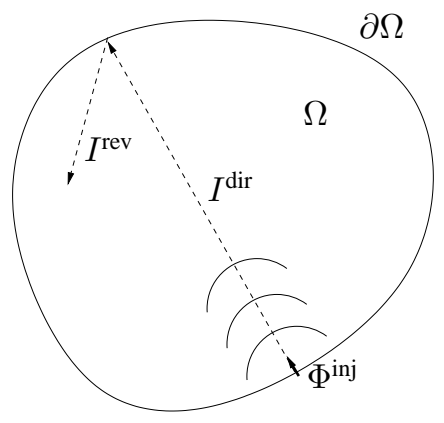

Figure 3: Considered system

- $\sigma_{i}(\omega)$ is the reverberated power flow;

- $\alpha_{i}(\omega)$ is the absorption coefficient.

The input power flow $\Phi_{i}^{\mathrm{inj}}(\omega)$ is injected in the cavity. In order to calculate $I$ and $W$ in the cavity, the directivity of these input power flows must be known. For high frequency, we assume the following directivity:

$$
d(\theta)=\frac{\cos \theta}{\pi}
$$

where $\theta$ is defined in figure 4. There is no way to evaluate the "correct" directivity through MES method, and the lambertian directivity $d(\theta)=\frac{\cos \theta}{\pi}$ is not always a good approximation. The hybrid method proposed in the paper should aim at considering the correct directivity to calculate the direct field.

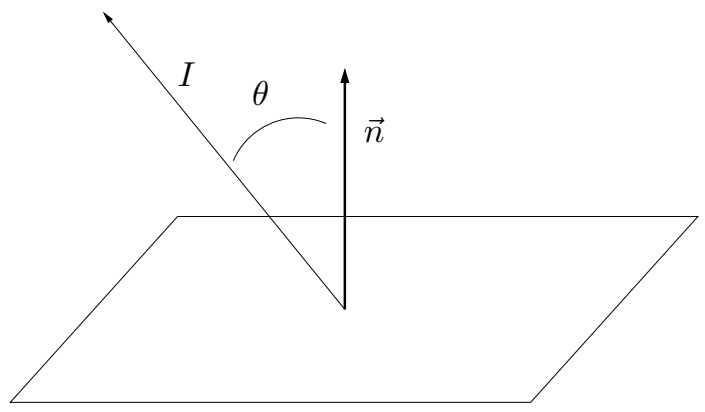

Figure 4: Notation for directivity

It is now possible to define $W^{\mathrm{dir}}(M)$ and $I^{\mathrm{dir}}(M)$ in function of $\Phi^{\text {inp }}$. Considering that $\eta<<1$ in the fluid, equation 13 leads to the following equations: 


$$
\begin{aligned}
W^{\mathrm{dir}}(M) & =\sum_{i=1}^{N_{e}} \frac{\Phi_{i}^{\mathrm{inp}}}{c_{0}} \iint_{S_{i}} \frac{d_{i}(\theta)}{r^{2}} d P \\
\vec{I}^{\mathrm{dir}}(M) & =\sum_{i=1}^{N_{e}} \Phi_{i}^{\mathrm{inp}} \iint_{S_{i}} \frac{d_{i}(\theta)}{r^{2}} \vec{u}_{r} d P
\end{aligned}
$$

where $r=\mathrm{PM}$ and $\vec{u}_{r}=\frac{\overrightarrow{M P}}{\|\overrightarrow{M P}\|}$ (see figure1, $S_{i}$ corrsponds to the facet number $i$. The reverberated field can be expressed the same way:

$$
\begin{aligned}
W^{\mathrm{rev}}(M) & =\sum_{i=1}^{N_{e}} \frac{\sigma_{i}^{\mathrm{inp}}}{c_{0}} \iint_{S_{i}} \frac{d_{i}(\theta)}{r^{2}} d P \\
\vec{I}^{\mathrm{rev}}(M) & =\sum_{i=1}^{N_{e}} \sigma_{i}^{\text {inp }} \iint_{S} \frac{d_{i}(\theta)}{r^{2}} \vec{u}_{r} d P
\end{aligned}
$$

Considering equations 14 and 15 , it is then possible to write $W$ and $\vec{I}$. In the following, we will consider $I=\vec{I} \cdot \vec{n}_{I}$ where $\vec{n}_{I}$ is a given vector, which corresponds to measurements of intensity among vector $\vec{n}_{I}$.

where $P$ is a point situated on an element $S_{i} . d_{i}(\theta)$ has been defined in equation 19 . $N_{e}$ is the number of elements.

Considering one element $i$, the power balance can be expressed as follows:

$$
\begin{aligned}
\sigma_{i}=\frac{1-\alpha_{i}}{S_{i}}\left[\sum_{\substack{j=1 \\
j \neq i}}^{N_{e}} \Phi_{j}^{\mathrm{inp}} \iint_{S_{i}} \iint_{S_{j}} \frac{\cos \theta_{i} d_{j}\left(\theta_{j}\right)}{r_{i j}^{2}} d P_{i} d P_{j}\right. \\
\\
\left.\quad+\sum_{\substack{j=1 \\
j \neq i}}^{N_{e}} \sigma_{j} \iint_{S_{i}} \iint_{S_{j}} \frac{\cos \theta_{i} d_{j}\left(\theta_{j}\right)}{r_{i j}^{2}} d P_{i} d P_{j}\right]
\end{aligned}
$$

This equation can be elaborated considering incoming and outcoming energy on the considered facet.

\subsection{Matrix equations}

Equation 24 can be written for $i=\left\{1,2, \ldots, N_{e}\right\}$ ( $N_{e}$ equations can be written). These equations can be expressed using matrices as follows:

$$
\mathbf{M} \sigma=\mathbf{B}
$$

with the following notations: 


$$
\begin{aligned}
\mathbf{M} & =\mathbf{I d}-(\mathbf{I d}-\mathbf{A}) \mathbf{T} \\
\mathbf{B} & =(\mathbf{I d}-\mathbf{A}) \mathbf{T} \boldsymbol{\Phi}^{\text {inp }}
\end{aligned}
$$

$\mathbf{T}$ and $\mathbf{A}$ are defined as follows:

$$
\begin{aligned}
T_{i j} & =\frac{1}{S_{j}} \iint_{S_{i}} \iint_{S_{j}} \frac{d_{i}\left(\theta_{i}\right) \cos \theta_{j}}{r_{i j}^{2}} d P_{i} d P_{j} \\
A_{i j} & =\delta_{i j} \alpha_{i}
\end{aligned}
$$

Notice that $\mathbf{T}$ must verify two properties, due to the conservation of energy:

$$
\begin{aligned}
& \sum_{j=1}^{N_{e}} T_{i j}=1 \quad \forall i \\
& \sum_{i=1}^{N_{e}} \frac{S_{i}}{S_{j}} T_{i j}=1 \quad \forall j
\end{aligned}
$$

Equation 25 allows to write the expression of $\sigma$ :

$$
\boldsymbol{\sigma}=\mathbf{M}^{-1} \mathbf{B}=\mathbf{M}^{-1}(\mathbf{I d}-\mathbf{A}) \mathbf{T} \boldsymbol{\Phi}^{\text {inp }}
$$

Direct and reverberated fields expressed in equations 20, 21, 22, and 23 can now be written as follows:

$$
\begin{aligned}
\mathbf{W} & =\mathbf{Y}^{W} \boldsymbol{\Phi}^{\text {inp }}+\mathbf{Y}^{W} \boldsymbol{\sigma} \\
\mathbf{I} & =\mathbf{Y}^{I} \boldsymbol{\Phi}^{\text {inp }}+\mathbf{Y}^{I} \boldsymbol{\sigma}
\end{aligned}
$$

Considering equation 32, $\mathbf{W}$ and $\mathbf{I}$ can be expressed as follow:

$$
\begin{gathered}
\mathbf{W}=\left\{\begin{array}{c}
W^{1} \\
W^{2} \\
\vdots \\
W^{N_{e}}
\end{array}\right\}=\mathbf{S}^{W} \boldsymbol{\Phi}^{\text {inp }} \\
\mathbf{I}=\left\{\begin{array}{c}
I^{1} \\
I^{2} \\
\vdots \\
I^{N_{e}}
\end{array}\right\}=\mathbf{S}^{I} \boldsymbol{\Phi}^{\text {inp }}
\end{gathered}
$$

where:

$$
\begin{aligned}
\mathbf{S}^{W} & =\mathbf{Y}^{W}\left[\mathbf{I d}+\mathbf{M}^{-1}(\mathbf{I d}-\mathbf{A}) \mathbf{T}\right] \\
\mathbf{S}^{I} & =\mathbf{Y}^{I}\left[\mathbf{I} \mathbf{d}+\mathbf{M}^{-1}(\mathbf{I} \mathbf{d}-\mathbf{A}) \mathbf{T}\right]
\end{aligned}
$$




\section{Hybrid Simplified Energy Method}

\subsection{Principle}

The main issue of the Simplified Energy Method is the unknown directivity of the sources $\Phi^{\text {inp }}$. In the previous section, it is assumed to be $d(\theta)=\frac{\cos \theta}{\pi}$ (see equation 19). The aim of the present section is to improve Simplified Energy Method in order to take into account the genuine directivity. Thus, Hybrid Simplified Energy Method (HMES) is based on a BEM calculation for the direct field. The reverberated field is calculated as for Simplified Energy Method, as shown in figure 5 .

The hybrid method allows to combine the advantages of MES and BEM. Indeed, calculating the entire problem through BEM would lead to very high calculation costs. That is why BEM will only be applied around the boundary sources.

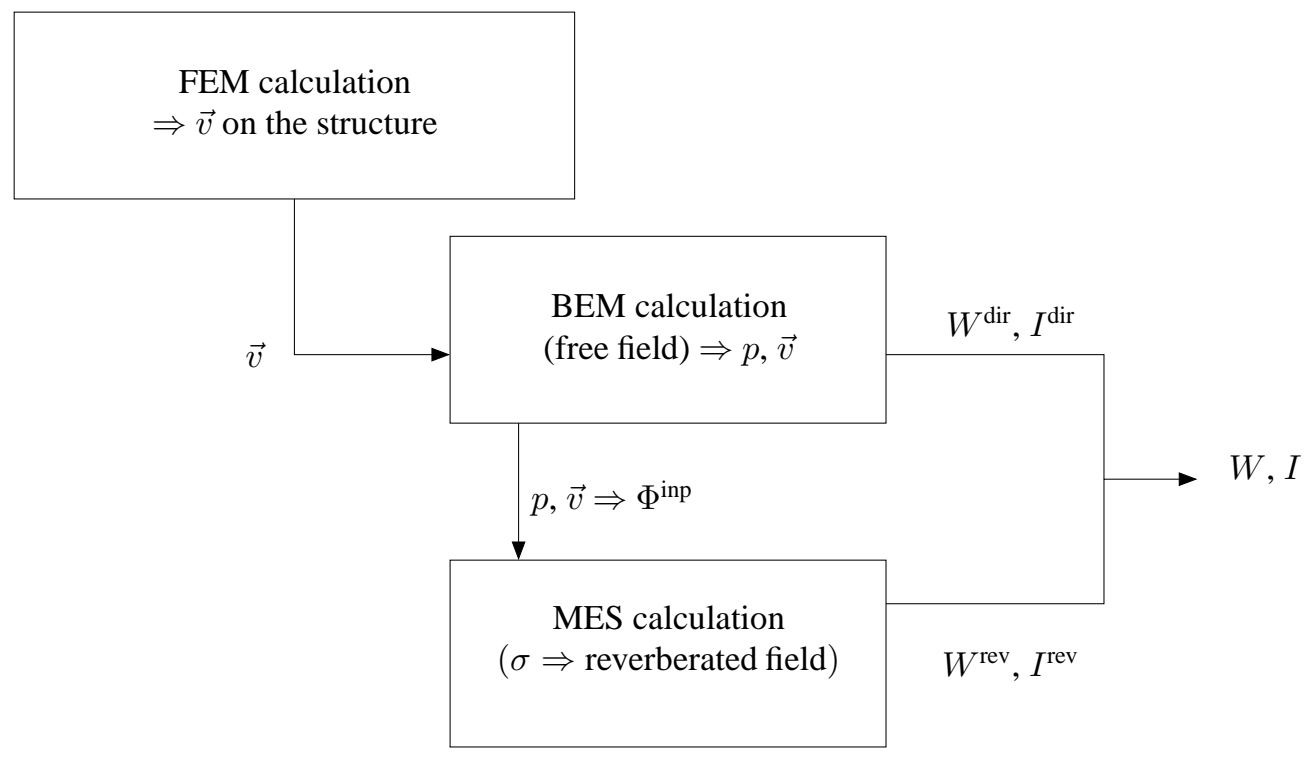

Figure 5: HMES method

The vibrating structure is first studied using a finite element code. The pressure and celerity fields on the structure is then computed in order to obtain the pressure field for the acoustic domain using a BEM code. Figure 6 shows the various fields considered in HMES. HMES is the computed using the fields denoted $I^{\mathrm{dir}}(\mathrm{BEM})$ and $I^{\mathrm{rev}}(\mathrm{MES})$ (an equivalent figure can of course be plot for $W$ fields):

$$
\begin{aligned}
I^{\mathrm{tot}}(\mathrm{HMES}) & =I^{\mathrm{dir}}(\mathrm{BEM})+I^{\mathrm{rev}}(\mathrm{MES}) \\
W^{\mathrm{tot}}(\mathrm{HMES}) & =W^{\mathrm{dir}}(\mathrm{BEM})+W^{\mathrm{rev}}(\mathrm{MES})
\end{aligned}
$$




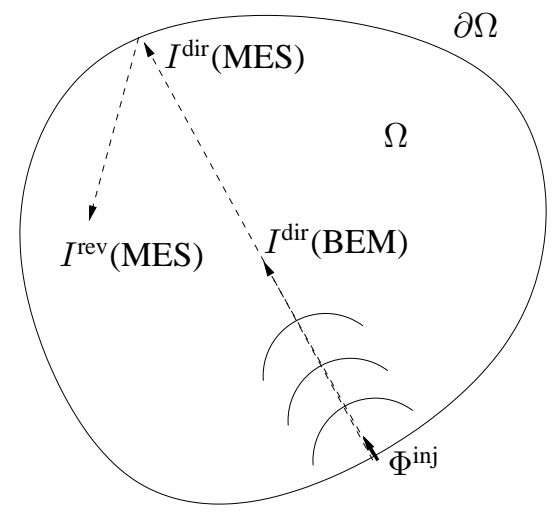

Figure 6: Considered system

\subsection{Direct field calculation}

The input power $\Phi^{\text {inp }}$ is due to a structure that is first studied using a FEM software. This calculation provides the celerity field on the structure. A BEM software is then used to obtain the pressure field on the structure, considering anechoic conditions. Thus, pressure and celerity are both known on the structure and it is possible to calculate the pressure field in the cavity, taking the directivity into account.

Let $\left(p_{I}, \vec{v}_{I}\right)$ be the pressure and celerity fields on the structure. These fields can be provided by any FEM software and a BEM calculation. A BEM calculation is then performed to obtain the pressure field $p(M)$ in the cavity:

$$
c p(M)=\int_{S}\left(p_{I}(P) \frac{\partial g}{\partial \vec{n}}-g(\overrightarrow{\mathrm{MP}}) \frac{\partial p_{I}}{\partial \vec{n}}\right) d P, \quad P \in S
$$

Notice that $\frac{\partial p_{I}}{\partial \vec{n}}=-\rho \omega \vec{v}_{I} \cdot \vec{n}$, where $\rho$ is the density of the fluid. $\vec{n}$ is the surface unit normal vector anc $c$ is defind as follows:

$$
c= \begin{cases}1, & \mathrm{M} \in \Omega \\ \frac{1}{2}, & \mathrm{M} \text { is on a smooth boundary of } \Omega \\ \frac{\mathcal{S}}{4 \pi}, & \mathrm{M} \text { is on a nonsmooth boundary of } \Omega\end{cases}
$$

where $\mathcal{S}$ is the solid angle. $g$ is the free-space green function defined as follows:

$$
g(\overrightarrow{\mathrm{MP}})=\frac{e^{-i k\|\overrightarrow{\mathrm{MP}}\|}}{4 \pi\|\overrightarrow{\mathrm{MP}}\|}
$$

The pressure field in the cavity allows to calculate the direct fields $W^{\mathrm{dir}}$ and $I^{\mathrm{dir}}$ : 


$$
\begin{aligned}
W^{\operatorname{dir}}(M) & =\frac{1}{2 \rho c^{2}} p(M) p^{*}(M) \\
I^{\operatorname{dir}}(M) & =\frac{1}{2} \Re\left[p(M) v^{*}(M)\right]
\end{aligned}
$$

$v=\vec{v} \cdot \vec{n}_{p}$ (and $v^{*}$ ) is obtained thanks to $p$ using equation $\frac{\partial p}{\partial \vec{n}_{p}}=-\rho \omega \vec{v} \cdot \vec{n}_{p}$ where $\vec{n}_{p}$ is a freely chosen unit vector. Thus $I$ corresponds to the projection of $\vec{I}$ among the direction $\vec{n}_{p}$.

\subsection{Reverberated field calculation}

The reverberated field is being calculated using classical MES theory described in section 2 The input power $\Phi^{\text {inp }}$ is provided by the pressure and celerity fields on the structure:

$$
\Phi^{\mathrm{inp}}=\frac{1}{2} \Re\left(p_{i} v_{i}^{*}\right)
$$

$\sigma$ is then calculated, which allows to obtain $W^{\text {rev }}$ and $I^{\text {rev }}$, as explained in equations 22 and 23

\subsection{Coupled matrix formulation}

Using a matrix formulation, the pressure computed by BEM can be written as follows:

$$
p_{I}=\mathbf{H}_{p} \mathbf{p}_{s t r}+\mathbf{G}_{p} \mathbf{v}_{s t r}
$$

where $\mathbf{H}_{p}$ and $\mathbf{G}_{p}$ are BEM matrices, and $\mathbf{p}_{s t r}$ and $\mathbf{v}_{\text {str }}$ are the pressure and celerity fields on the structure (computed by a finite element code). An equivalent equation can be written for the celerity field:

$$
v_{I}=\mathbf{H}_{v} \mathbf{p}_{s t r}+\mathbf{G}_{v} \mathbf{v}_{\text {str }}
$$

Thanks to equations 44 and 45] equations 47 and 48 lead to the following equations concerning the direct field:

$$
\begin{aligned}
W_{n}^{\mathrm{dir}} & =\frac{1}{2 \rho c^{2}}\left(\begin{array}{ll}
\mathbf{p}_{s t r}^{*} & \mathbf{v}_{s t r}^{*}
\end{array}\right) \boldsymbol{\Lambda}_{1}\left(\begin{array}{c}
\mathbf{p}_{s t r} \\
\mathbf{v}_{s t r}
\end{array}\right) \\
I_{n}^{\mathrm{dir}} & =\frac{1}{2} \Re\left[\left(\begin{array}{ll}
\mathbf{p}_{s t r}^{*} & \mathbf{v}_{s t r}^{*}
\end{array}\right) \boldsymbol{\Lambda}_{2}\left(\begin{array}{c}
\mathbf{p}_{s t r} \\
\mathbf{v}_{s t r}
\end{array}\right)\right]
\end{aligned}
$$

where $W_{n}^{\mathrm{dir}}$ is the $\mathrm{n}^{\text {th }}$ value of $\mathbf{W}^{\mathrm{dir}}$ and $I_{n}^{\mathrm{dir}}$ is the $\mathrm{n}^{\text {th }}$ value of $\mathbf{I}^{\mathrm{dir}} . \Lambda_{1}$ and $\Lambda_{2}$ are defined as follows: 


$$
\begin{aligned}
& \Lambda_{1}=\left[\begin{array}{l|l}
\mathbf{H}_{p}^{*} \mathbf{H}_{p} & \mathbf{H}_{p}^{*} \mathbf{G}_{p} \\
\hline \mathbf{G}_{p}^{*} \mathbf{H}_{p} & \mathbf{G}_{p}^{*} \mathbf{G}_{p}
\end{array}\right] \\
& \Lambda_{2}=\left[\begin{array}{l|l}
\mathbf{H}_{p}^{*} \mathbf{H}_{v} & \mathbf{H}_{p}^{*} \mathbf{G}_{v} \\
\hline \mathbf{G}_{p}^{*} \mathbf{H}_{v} & \mathbf{G}_{p}^{*} \mathbf{G}_{v}
\end{array}\right]
\end{aligned}
$$

Concerning the reverberated field, $\mathbf{W}^{\text {rev }}$ and $\mathbf{I}^{\text {rev }}$ are obtained using equations 35 and 36

$$
\begin{aligned}
W_{n}^{\mathrm{rev}} & =\frac{1}{2} \mathbf{S}_{n}^{W} \Re\left[\left(\mathbf{p v}^{*}\right)_{s t r}\right] \\
I_{n}^{\mathrm{rev}} & =\frac{1}{2} \mathbf{S}_{n}^{I} \Re\left[\left(\mathbf{p v}^{*}\right)_{s t r}\right]
\end{aligned}
$$

where $\mathbf{S}_{n}^{W}$ is the $\mathrm{n}^{\text {th }}$ line of matrix $\mathbf{S}_{n}^{W}$ and $\mathbf{S}_{n}^{I}$ is the $\mathrm{n}^{\text {th }}$ line of matrix $\mathbf{S}_{n}^{I}$. $W^{\text {tot }}$ and $I^{\text {tot }}$ can then be written:

$$
\begin{aligned}
W_{n}^{\mathrm{tot}} & =\left(\begin{array}{ll}
\mathbf{p}_{s t r}^{*} & \mathbf{v}_{s t r}^{*}
\end{array}\right) \tilde{\mathbf{\Lambda}}_{1}\left(\begin{array}{c}
\mathbf{p}_{s t r} \\
\mathbf{v}_{s t r}
\end{array}\right) \\
I_{n}^{\mathrm{tot}} & =\Re\left[\left(\begin{array}{ll}
\mathbf{p}_{s t r}^{*} & \mathbf{v}_{s t r}^{*}
\end{array}\right) \tilde{\mathbf{\Lambda}}_{2}\left(\begin{array}{c}
\mathbf{p}_{s t r} \\
\mathbf{v}_{s t r}
\end{array}\right)\right]
\end{aligned}
$$

where $\tilde{\Lambda}_{1}$ and $\tilde{\Lambda}_{2}$ are defined as follows:

$$
\begin{aligned}
\tilde{\Lambda}_{1} & =\frac{1}{2 \rho c^{2}}\left[\begin{array}{c|c|c}
\mathbf{H}_{p}^{*} \mathbf{H}_{p} & \mathbf{H}_{p}^{*} \mathbf{G}_{p} \\
\hline \mathbf{G}_{p}^{*} \mathbf{H}_{p} & \mathbf{G}_{p}^{*} \mathbf{G}_{p}
\end{array}\right]+\frac{1}{4}\left[\begin{array}{c|c}
0 & \operatorname{diag}\left\{\mathbf{S}_{n}^{W}\right\} \\
\hline \operatorname{diag}\left\{\mathbf{S}_{n}^{W}\right\} & 0
\end{array}\right] \\
\tilde{\Lambda}_{2} & =\frac{1}{2}\left[\begin{array}{l|l}
\mathbf{H}_{p}^{*} \mathbf{H}_{v} & \mathbf{H}_{p}^{*} \mathbf{G}_{v} \\
\hline \mathbf{G}_{p}^{*} \mathbf{H}_{v} & \mathbf{G}_{p}^{*} \mathbf{G}_{v}
\end{array}\right]+\frac{1}{4}\left[\begin{array}{c|c}
0 & \operatorname{diag}\left\{\mathbf{S}_{n}^{I}\right\} \\
\hline \operatorname{diag}\left\{\mathbf{S}_{n}^{I}\right\} & 0
\end{array}\right]
\end{aligned}
$$

\section{Results}

The structure and the cavity used for the examples are shown on figure 7 Only one panel is able to move (the "structure"). The others are considered to be infinitely rigid (i.e. $\overrightarrow{g r a d} p=0$ ). The calculations have been made using MATLAB and OPENBEM [37]. Of course, the meshing of the structure has to be quite fine in order to provide good FEM and BEM results. On the contrary, the MES calculation does not need fine meshes.

$W$ is calculated using classical MES (figure 8 ) and the method we propose (figure 9. Concerning the BEM calculation, a frequency average has been done. The data used for this calculation are recalled in table 2

Notice that the dimensions of the box allow to justify the mid-high frequency range situation. Indeed, the acoustic modes are situated at the following frequencies: 


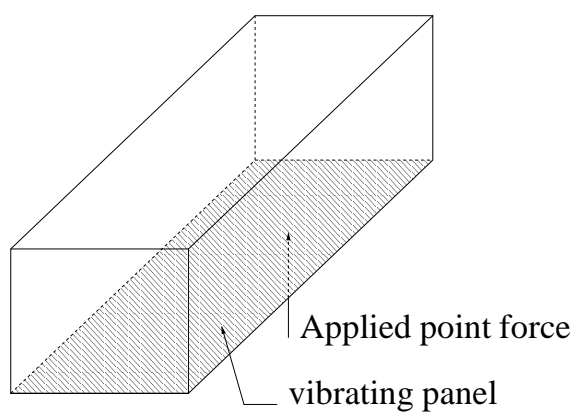

Figure 7: Structure used for the calculations

$$
f=\frac{c}{2} \sqrt{\left(\frac{n_{x}}{L_{x}}\right)^{2}+\left(\frac{n_{y}}{L_{y}}\right)^{2}+\left(\frac{n_{z}}{L_{z}}\right)^{2}}
$$

The modal density around $f=2000 \mathrm{~Hz}$ can then be evaluated to $d=30$ modes $/ \mathrm{Hz}$, which is enough to consider high frequency domain.

Figure 10 show a BEM calculation taking into account the impedance on the boundaries. This calculation is considered to be "exact". Considering these results, it appears that HMES gives much better results that MES: taking into account the right directivity of the source allows to provide more accurate results. MES remains quite efficient if only the $W$ average in the cavity is considered, but locally results are far from "exact" results (BEM results), especially around the source point.

Concerning the calculation costs, table 1 shows the differences between MES, BEM and the hybrid method.

\begin{tabular}{|c|c|c|c|}
\hline$\overline{\text { Method }}$ & Number of elements & Calculation cost & Error (\% vs BEM method) \\
\hline MES & around 100 & a few minutes & $\begin{array}{l}\text { around } 10 \% \text { in front of the source } \\
50-200 \% \text { far from the source }\end{array}$ \\
\hline BEM & more than 5000 & 2 days & reference \\
\hline Hybrid method & around 500 & around 15 minutes & $\begin{array}{l}\text { around } 10 \% \text { in front of the source } \\
10-40 \% \text { far from the source }\end{array}$ \\
\hline
\end{tabular}

Table 1: Comparison between the methods - calculation costs

\begin{tabular}{|c|c|c|c|c|}
\hline \hline$\rho$ & $c$ & Dimensions & Frequency & $\alpha$ (absoption coefficient) \\
\hline $1,25 \mathrm{kgm}^{-3}$ & $340 \mathrm{~ms}^{-1}$ & $2 \times 2 \times 4 \mathrm{~m}^{3}$ & $1500-2500 \mathrm{~Hz}$ & 0,3 \\
\hline \hline
\end{tabular}

Table 2: Data used for calculations 


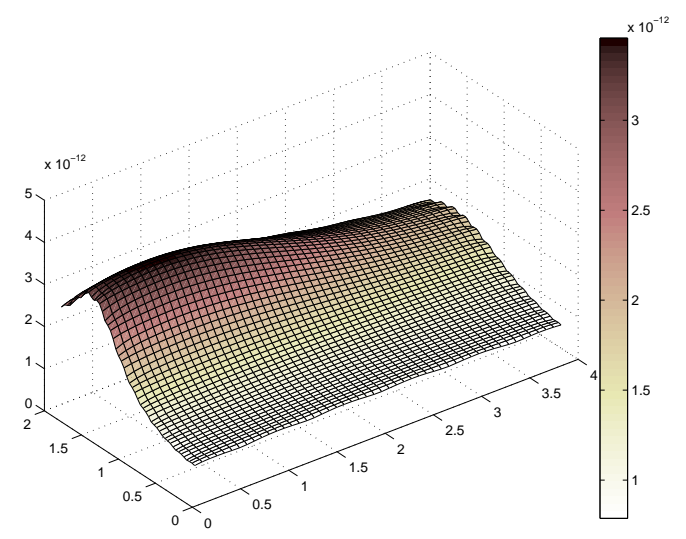

Figure 8: $W$ computed by MES

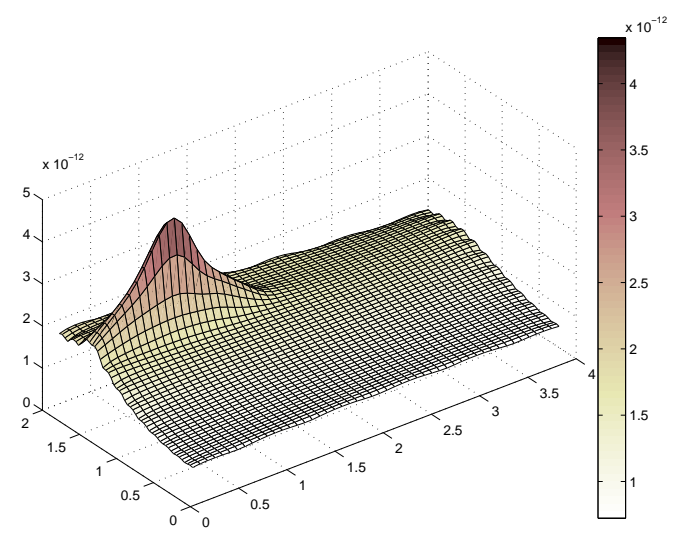

Figure 9: $W$ computed by HMES

\section{Conclusion}

The method proposed in this paper allows to predict the acoustical behaviour of a cavity. This energy method is more precise that existing methods like MES or SEA because it is able to take into account the directivity of the sources by FEM/BEM calculations without meshing the cavity. Moreover, it is possible to take the damping into account, even if it is not low. Compared to a full BEM or FEM calculation, the method we propose only requires finer meshing around the sources. The other parts of the cavity do not need finer meshing. The advantages and disadvantages of the method are 


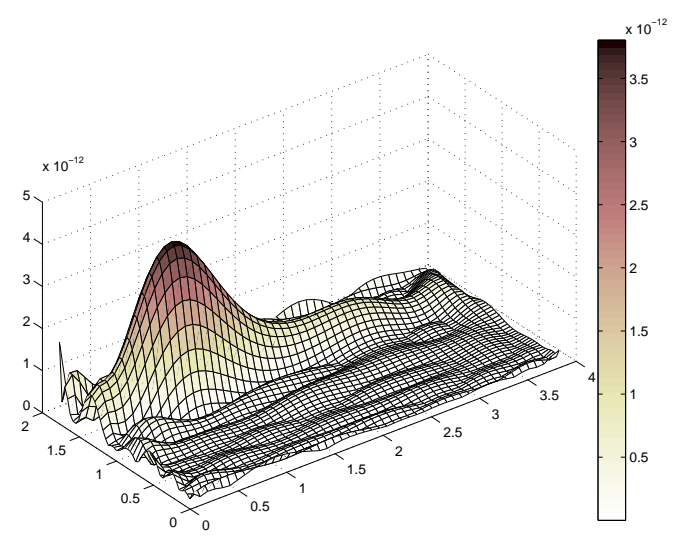

Figure 10: $W$ computed by BEM

given in table 3

\begin{tabular}{|l|l|l|}
\hline \hline Method & \multicolumn{1}{|c|}{ Advantages } & \multicolumn{1}{c|}{ Disadvantages } \\
\hline \hline \multirow{2}{*}{ BEM } & $\begin{array}{l}\text { - More accurate calculations } \\
- \text { No 3D meshing }\end{array}$ & $\begin{array}{l}\text { - Finer 2D meshing } \\
- \text { Full matrices } \\
- \text { Very big matrices in high frequency }\end{array}$ \\
\hline \multirow{2}{*}{ FEM } & $\begin{array}{l}\text { - More accurate calculations } \\
- \text { Sparse matrices }\end{array}$ & $\begin{array}{l}\text { - Finer 2D and 3D meshing } \\
- \text { Very big matrices in high frequency }\end{array}$ \\
\hline \multirow{2}{*}{ MES } & $\begin{array}{l}\text { - Light calculations } \\
\text { - Height speed even in high frequency }\end{array}$ & $\begin{array}{l}\text { - Directivity badly taken into account } \\
\text { - Not valid in low frequency }\end{array}$ \\
\hline \multirow{2}{*}{ HMES } & $\begin{array}{l}\text { - Light calculations } \\
\text { - Height speed even in high frequency } \\
\text { - Directivity taken into account (direct field) }\end{array}$ & $\begin{array}{l}\text { - Directivity badly taken into account (reverberated field) } \\
- \text { Not valid in low frequency }\end{array}$ \\
\hline
\end{tabular}

Table 3: Advantages / Disadvantages of HMES

The results show that the total field is improved, even in a simple case of a box as presented in figure 7 Nevertheless, the calculations are longer than for the MES method because of the FEM and BEM calculations. Moreover, unlike MES method, the BEM need quite fine meshes and several calculation because of the frequency average.

\section{Acknowledgement}

This work was made within the framework of the European project CREDO. The authors thank all the partners of this project. 


\section{References}

[1] S. Besset and L. Jézéquel, Dynamic Substructuring based on a Double Modal Analysis, J. Vib. Acoust, 130 (1), 2008

[2] S. Besset and L. Jézéquel, Vibroacoustical Analysis Based on a Multimodal Strategy: Triple Modal Synthesis, J. Vib. Acoust, 130 (3), 2008

[3] R. H. Lyon, Statistical Energy Analysis of Dynamical Systems: Theory and Application, Cambridge, Massachusetts, MIT Press, 1975.

[4] D. Nefske, S. Sung, Power flow finite element analysis of dynamic systems: Basic theory and application to beams, NCA Publication 3.

[5] J. Wohlever, R. Bernhard, Mechanical energy flow models of rods and beams, Journal of Sound and Vibration 153 (1992) 1-19.

[6] O. Bouthier, R. Bernhard, Simple models of energy flow in vibrating membranes, Journal of Sound and Vibration 182 (1995) 129-147.

[7] O. Bouthier, R. Bernhard, Simple models of energy flow in vibrating plates, Journal of Sound and Vibration 182 (1995) 149-164.

[8] Y. Lase, M. Ichchou, L. Jezequel, Energy analysis of bars an beams: Theoretical formulations, Journal of Sound and Vibration 192 (1996) 281-305.

[9] A. Le Bot, Equations énergétiques en mécanique vibratoire: application au domaine des moyennes et hautes fréquences, Ph.D. thesis, Ecole Centrale de Lyon, 94-20 (1994).

[10] M. N. Ichchou, Formulations énergétiques pour l'étude moyennes et hautes fréquences des systèmes : théorie et applications, Ph.D. thesis, Ecole Centrale de Lyon, 96-10 (1996).

[11] R. Langley, On the vibrational conductivity approach to high frequency dynamics for two-dimensional structural components, Journal of Sound and Vibration 182 (1995) 637-657.

[12] A. Carcaterra, A. Sestieri, Energy density equations and power flow in structures, Journal of Sound and Vibration 188 (1995) 269-282.

[13] A. Carcaterra, Wavelength scale effects on energy propagation in structures, IUTAM Symposium on Statistical Energy Analysis Soutampton, UK 8-11 July 1997 (Kluwer, 1998) ed F. J. Fahy and W. G. Price.

[14] J. T. Xing, W. G. Price, A power flow analysis based on continum dynamics, Proc. London Royal Society A (455) (1999) 401-436.

[15] P. Moron, Mise en œuvre de méthodes énergétiques dans létude du comportement vibratoire de structures complexes dans le domaine des moyennes et hautes fréquences, Ph.D. thesis, Ecole Centrale de Lyon, 98-16 (1998). 
[16] L. Houillon, Modélisation vibratoire des carrosseries automobiles en moyennes et hautes fréquences, Ph.D. thesis, Ecole Centrale de Lyon, 99-49 (1999).

[17] A. Bocquillet, Méthodes énergétiques de caractérisations vibroacoustiques des réseaux complexes, Ph.D. thesis, Ecole Centrale de Lyon, 00-01 (2000).

[18] A. Wang, N. Vlahopoulos, K. Wu, Development of an energy boundary element formulation for computing high-frequency sound radiation from incoherent intensity boundary conditions, Journal of Sound and Vibration.

[19] O. M. Bouthier, R. J. Bernhard, Simple models of energy flow in vibrating membranes, Journal of Sound and Vibration 182 (1) (1995) 129-147.

[20] O. M. Bouthier, R. J. Bernhard, Simple models of the energetics of transversely vibrating plates, Journal of Sound and Vibration 182 (1) (1995) 149-164.

[21] V. Cotoni, Modélisation de phénomènes vibroacoustiques en moyenne et hautes fréquences par méthode énergétique locale, Ph.D. thesis, Ecole Centrale de Lyon (2001).

[22] V. Cotoni, A. Le Bot, L. Jezequel, High frequency radiation of 1-shaped plates by a local energy flow approach, Journal of Sound and Vibration 250 (3) (2002) 431-444.

[23] P. Hardy, L. Jezequel, M. Ichchou, Absorption coefficient and energy flow path identification by means of inverse local energy method, The Journal of the Acoustical Society of America 112 (5) (2002) 2320-2320.

[24] P. Shorter, R. Langley, Vibro-acoustic analysis of complex systemsr. s., Journal of Sound and Vibration 288 (2005) 669-699.

[25] L. Brillouin, Wave propagation in periodic structures, Dover Publications,Inc.,New York, 1953.

[26] K. Morse, H. Feshbach, Methods of theoretical physics, Mac Graw Hill, New York, 1953.

[27] M. Ichchou, L. Jezequel, Comments on simple models of the energy flow in vibrating membranes and transversely vibrating plates, Journal of Sound and Vibration 195 (1996) 679-685.

[28] M. J. Smith, A hybrid energy method for predicting vibrational response of point loaded plates, Journal of Sound and Vibration 202 (1997) 375-392.

[29] M. Ichchou, L. Jezequel, Transient local energy approach for multi-dimensional, a review with extensions, Submitted to Journal of Acoustical Society of America.

[30] H. Kuttruff, Simulierte nachhallkurven in rechteckräumen mit diffusem schallfeld, Acoustica 25 (1971) 333-342. 
[31] H. Kuttruff, A simple iteration scheme for the computation of decay constants in enclosures with diffusely reflecting boundaries, Journal of Acoustical Society of America 98(1) (1995) 288-293.

[32] H. Kuttruff, Room acoustics, 3rd Edition, Elsevier, 1991.

[33] H. Kuttruff, Energetic sound propagation in rooms, Acoustica with Acta Acoustica 83 (1997) 622-628.

[34] M. M. Carroll, C. F. Chien, Decay of reverberent sound in a spherical enclosure, Journal of Acoustical Society of America 62(6) (1977) 1442-1446.

[35] R. N. Miles, Sound field in a rectangular enclosure with diffusely reflecting boundaries, Journal of Sound and Vibration 92(2) (1984) 203-226.

[36] A. Le Bot, A vibroacoustic model for high frequency analysis, Journal of Sound and Vibration 211(4) (1998) 537-654.

[37] P. Juhl, The boundary element method for sound field calculations, Ph.D. thesis, Technical University of Denmark (1993). 